\title{
Symmetry Groups and Non-Abelian Cohomology
}

\author{
R. F. Streater* \\ Center for Transport Theory and Mathematical Physics, Department of Mathematics and \\ Physics, Virginia Polytechnic Institute, Blacksburg, VA 24061, USA
}

\section{Dedicated to Res Jost and Arthur Wightman}

\begin{abstract}
We consider the implementation of symmetry groups of automorphisms of an algebra of observables in a reducible representation whose multipliers in general are non-commuting operators in the commutant of the representation. The multipliers obey a non-abelian cocycle relation which generalizes the 2-cohomology of the group. Examples are given from the theory of spin algebras and continuous tensor products. For type $I$ representations we show that the multiplier can be chosen to lie in the centre, giving an isomorphism with abelian theory.
\end{abstract}

\section{Introduction}

We start with Wigner's formulation of symmetry in quantum mechanics [1], which was used with serene power for the Poincare group $\mathbb{P}_{+}^{\uparrow}[2]$. The states $\{\boldsymbol{\psi}\}$ of a system are taken to form the unit sphere $\mathscr{H}_{1}$ in a projective Hilbert space $\mathscr{H}$ : so if $\mathscr{H}$ is a Hilbert space and $\psi \in \mathscr{H}$ with $\|\psi\|=1$, then the state $\psi$ is the unit ray through $\psi$ :

$$
\boldsymbol{\psi}=\{\lambda \psi:|\lambda|=1, \lambda \in \mathbb{C}\} \dot{\epsilon} \mathscr{H}_{1} .
$$

We furnish $\mathscr{H}_{1}$ with a transition probability:

$$
\mathscr{P}(\boldsymbol{\psi}, \boldsymbol{\phi})=|\langle\psi, \phi\rangle|^{2}, \quad \psi \in \boldsymbol{\psi}, \phi \in \boldsymbol{\phi} .
$$

A symmetry operation is an isometry $\mathbf{U}$, that is, a bijection $\mathbf{U}: \mathscr{H}_{1} \rightarrow \mathscr{H}_{1}$ preserving $\mathscr{P}$ :

$$
\mathscr{P}(\mathbf{U} \boldsymbol{\psi}, \mathbf{U} \gamma)=\mathscr{P}(\boldsymbol{\psi}, \boldsymbol{\phi}) \text { for all } \boldsymbol{\psi}, \boldsymbol{\phi} \in \mathscr{H}_{1} \text {. }
$$

The set of isometries is a group, denoted Aut $\mathscr{H}_{1}$.

* Permanent address: Department of Mathematics, King's College, Strand, London WC2R2LS, UK 
If we have a group of symmetries, $G$, we obtain a projective representation of $G$, i.e. a homomorphism $\mathbf{U}: G \rightarrow$ Aut $\mathscr{H}_{1}$. Each isometry $\mathbf{U}(\mathrm{g})$ can be implemented by a unitary or anti-unitary operator $U_{g}$ on $\mathscr{H}$ [3], which is ambiguous up to a phase, i.e. an element of $U(1)$. Let us take the unitary case. Then the map $g \rightarrow U_{g}$ is a multiplier representation:

$$
U_{g} U_{h}=\omega(g, h) U_{g h}, \quad g, h \in G,
$$

where $\omega(g, h) \in U(1)$ is the multiplier. The associativity of $U_{g}, U_{h}, U_{k}$ leads to the cocycle relation

$$
\omega(g, h) \omega(g h, k)=\omega(h, k) \omega(g, h k), \quad g, h, k \in G .
$$

If $U_{g}$ is replaced by

$$
U_{g}^{\prime}=\mu(g) U_{g}, \quad \mu(g) \in U(1),
$$

we do not change the projective isometry: $\mathbf{U}_{g}=\mathbf{U}_{g}^{\prime}$. The multiplier $\omega^{\prime}$ for $U^{\prime}$ is related to $\omega$ by

$$
\omega^{\prime}(g, h)=\mu(g) \mu(h) \mu(g h)^{-1} \omega(g, h)
$$

and we say $\omega$ and $\omega^{\prime}$ are equivalent if (1.6) holds for some map $\mu: G \rightarrow U(1)$.

A cocycle of the form

$$
\omega_{0}(g, h)=\mu(g) \mu(h) \mu(g h)^{-1}
$$

is called trivial, or a coboundary. Using point-wise multiplication in $U(1)$, the set of cocycles form a group $Z^{2}$ and the set of coboundaries form a subgroup $B^{2}$.

The second cohomology group, $H^{2}(G, U(1))$, defined as the quotient $Z^{2} / B^{2}$, describes the collection of possible inequivalent multipliers [4]. If $G$ is locally compact there do exist irreducible representations with each multiplier, since these can be obtained from the true representations of an extension of $G$ by $U(1)$.

If two representations of a group belong to inequivalent multipliers, then the states of one representation space cannot be coherently mixed with the states of the other - a superselection rule exists between the spaces. Examples are the univalence superselection rule which prevents the mixing of states of integer and non-integer spin, and Bargmann's superselection rule, which prevents the mixing of states of different mass in a Galileo-invariant theory. Without Wigner's idea of allowing multipliers, and superselection rules, we could not have particles of nonintegral spin, or the Heisenberg commutation relations between position and momentum in a Galilean invariant theory [5].

Wightman's hypothesis [6], that the superselection operators commute among themselves, leads to a slightly more general analysis. In Sect. 2 we consider a further generalization, non-abelian superselection rules. We show that in the type $I$ case we can reduce the non-abelian cocycles to abelian cocycles with left-action.

Wigner's approach is not designed to cope with spontaneously broken symmetry. In the BCS model [7] the Lagrangian (or Hamiltonian) is invariant under a group $G$ of transformations (the gauge group in this case), but the ground state is not invariant under the action of $G$. This idea was used by Nambu and Jona-Lasinio, and Goldstone [8] in theories of elementary particles, and is now known as a spontaneously broken symmetry. This is not a very precise criterion, 
since it leaves open the question as to whether or not the broken symmetry is given by a unitary (or anti-unitary) operator.

This question can be formulated more exactly in the $C^{*}$-algebra approach to quantum mechanics: a spontaneously broken symmetry is an automorphism of the $C^{*}$-algebra $\mathscr{A}$ of observables, commuting with the dynamics, but which is not a spatial automorphism in the representation in question [9]. Apart from its more precise formulation, avoiding Lagrangians, this allows us to consider representations without ground states.

Let $\left\{\pi_{q}\right\}$ be a collection of inequivalent irreducible representations of the algebra of observables, $\mathscr{A}$, on Hilbert spaces $\mathscr{H}_{q}$; the vectors of the $\mathscr{H}_{q}$ are possible states of the system. We can form the direct sum $\pi=\bigoplus_{q} \pi_{q}$ acting on $\mathscr{H}=\bigoplus_{q} \mathscr{H}_{q}$, with superselection rules operating between various $\mathscr{H}_{q}$. As constructed, $\pi$ is multiplicitly free. This is mathematically equivalent to the fact that the commutant $\pi(\mathscr{A})^{\prime}$ is abelian - Wightman's hypothesis. Thus, this hypothesis can be "justified" by asserting that, to get all possible states in $\mathscr{H}$, we only need to include the vector states of each $\mathscr{H}_{q}$ once each; there is no possible physical measurement that can distinguish between repeated copies.

In spite of this devastating argument, non-abelian gauge theories remain popular: these theories use repeated states (of different "colour") all having the same physics. It is therefore worthwhile to rethink the Wigner analysis in representations of $\mathscr{A}$ in which $\pi(\mathscr{A})^{\prime}$ is not abelian, and in which the multipliers $\omega(g, h)$ [unitary elements in $\pi(\mathscr{A})^{\prime}$ ] do not commute with the unitary operators $\left\{U_{g}, g \in G\right\}$ implementing the symmetries. This leads us to "non-abelian cohomology," outlined in Sect. 2. The main result is Theorem 1: if $\pi(\mathscr{A})$ is of type $I$, then there exists a family of gauge group elements, $\{\mu(g)\}_{g \in G} \in \pi(\mathscr{A})^{\prime}$, such that $U^{\prime}(g)$ $=\mu(g) U(g)\left[\right.$ cf. (1.6)] has multipliers $\omega^{\prime}(g, h)=U_{g}^{\prime} U_{h}^{\prime} U_{g h}^{\prime-1}$ lying in $\mathscr{Z}=\pi(\mathscr{A})^{\prime \prime}$ $\cap \pi(\mathscr{A})^{\prime}$, the centre of $\pi(\mathscr{A})^{\prime \prime}$, on which $G$ acts by left action; in short, $\omega^{\prime}$ is an abelian cocycle [4]. Moreover, equivalent non-abelian cocycles correspond to equivalent abelian ones.

Our general analysis can give rise to a new mechanism for breaking a symmetry, called "fact violation" in [10]. When a non-trivial multiplier occurs between a 1-parameter symmetry group and the time-evolution group, then the generator of the symmetry does not commute with the Hamiltonian $H$, and is thus not a conserved quantity, even though Wigner's isometric maps exist. This phenomenon is usually called an anomaly. In [10], the unitary operators representing space-translation do not commute. Indeed, $\mathbb{R}^{4}$ as a group has many multipliers; these are not used in relativistic theory [2], since they cannot be extended to multipliers of $\mathbb{P}_{+}^{\uparrow}$. This argument has no force in a non-relativistic model, or in a representation of a relativistic model in which the Lorentz group is broken (such as the charged sectors of QED). In Sect. 3 we give an example of a spin system in which the space-time automorphisms of $\mathscr{A}$ commute, but their implementing unitaries do not - a classic case of anomaly.

In Sect. 4 we give a continuous version of this model; we find that the multiplier is an operator on which the symmetry group acts non-trivially. That is, we get an operator anomaly. We also show, by this example, that it is possible for a gauge group, which by definition acts trivially on observables, to acquire an anomaly and be non-trivially implemented! 


\section{Non-Abelian Cocycles}

Let $\mathscr{A}$ be a $C^{*}$-algebra with unit, represented on the Hilbert space $\mathscr{H}$ by a representation $\pi: \mathscr{A} \rightarrow \mathbb{B}(\mathscr{H})=C^{*}$-algebra of all bounded operators on $\mathscr{H}$. Let $M=\pi(\mathscr{A})^{\prime}$, a possibly non-abelian $W^{*}$-algebra. Let $G$ be a group of automorphisms $\left\{\tau_{g}: g \in G\right\}$ of $\mathscr{A}$, each being spatial in $\pi$. That is, $\tau_{g}$ is implemented by $U_{g} \in$ Aut $\mathscr{H}$, thus:

$$
\pi\left(\tau_{g} A\right)=U_{g} \pi(A) U_{g}^{-1}, \quad g \in G, A \in \mathscr{A} .
$$

For any $V \in$ Aut $\mathscr{H}$, denote the adjoint action of $V$ on $\mathbb{B}(\mathscr{H})$ by $\operatorname{Ad} V$ :

$$
\operatorname{Ad} V B=V B V^{-1}, \quad B \in \mathbb{B}(\mathscr{H}) .
$$

Thus, (2.1) can be written

$$
\operatorname{Ad} U_{g} \pi(A)=\pi\left(\tau_{g} A\right), \quad g \in G, A \in \mathscr{A} .
$$

The map $g \rightarrow U_{g}$ need not be a group homomorphism, $G \rightarrow$ Aut $\mathscr{H}$; all we can say is that

$$
\omega(g, h) \stackrel{\text { def }}{=} U_{g} U_{h} U_{g h}^{-1} \in M
$$

Indeed, for $A \in \mathscr{A}$,

$$
\begin{aligned}
\omega(g, h) \pi(A)-\pi(A) \omega(g, h) & =(\operatorname{Ad} \omega(g, h) \pi(A)-\pi(A)) \omega(g, h) \\
& =\left(\operatorname{Ad} U_{g} \circ \operatorname{Ad} U_{h} \circ \operatorname{Ad} U_{g h^{-1}} \pi(A)-\pi(A)\right) \omega(g, h) \\
& =\left(\pi\left(\tau_{g} \circ \tau_{h} \circ \tau_{g h}^{-1} A\right)-\pi(A)\right) \omega(g, h) \\
& =0,
\end{aligned}
$$

showing that $\omega(g, h) \in M$. Hence we get the non-abelian multiplier representation

$$
U_{g} U_{h}=\omega(g, h) U_{g h}, \quad \omega \in M, g, h \in G .
$$

Now we show that $\operatorname{Ad} U_{g}$ maps $M$ to itself; indeed if $B \in M$ and $A \in \mathscr{A}$,

$$
\begin{aligned}
\operatorname{Ad} U_{g} B \pi(A)-\pi(A) \operatorname{Ad} U_{g} B & =U_{g}\left\{B U_{g}^{-1} \pi(A) U_{g}-U_{g}^{-1} \pi(A) U_{g} B\right\} U_{g}^{-1} \\
& =\operatorname{Ad} U_{g}\left[B, \pi\left(\tau_{g^{-1}} A\right)\right]=0 \text { for all } A \in \mathscr{A},
\end{aligned}
$$

showing $\operatorname{Ad}_{g} B \in M$ if $B \in M$.

A similar argument shows that $\operatorname{Ad} U_{g}$ maps $M^{\prime}=\pi(\mathscr{A})^{\prime \prime}$ to itself; or, $\operatorname{Ad} U_{g} \mid \pi(\mathscr{A})$, being (ultra)strongly continuous, has a unique extension to the closure, $\pi(\mathscr{A})^{\prime \prime}$, and this extension must be $\operatorname{Ad} U_{g} \mid \pi(\mathscr{A})^{\prime \prime}$. It follows from these results that $\operatorname{Ad} U_{g}$ maps $\mathscr{Z}=M \cap M^{\prime}$ to itself.

Whereas the map $g \rightarrow \operatorname{Ad} U_{g} \mid M^{\prime}$ is a group homomorphism, $G \rightarrow \operatorname{Aut} M^{\prime}$, it is not true in general that the map $\mathrm{g} \rightarrow \operatorname{Ad} U_{g} \mid M$ is a group homomorphism, $G \rightarrow \operatorname{Aut} M$.

Indeed, if $B \in \mathbb{B}(\mathscr{H})$,

$$
\begin{aligned}
\operatorname{Ad} U_{g} \circ \operatorname{Ad} U_{h} B & =U_{g} U_{h} B U_{h .}^{-1} U_{g}^{-1} \\
& =\omega(g, h) U_{g h} B U_{g h^{1}} \omega(g, h)^{-1} \\
& =\operatorname{Ad} \omega(g, h) \circ \operatorname{Ad} U_{g h} B
\end{aligned}
$$


so

$$
\operatorname{Ad} U_{g} \circ \operatorname{Ad} U_{h}=\operatorname{Ad} \omega(g, h) \circ \operatorname{Ad} U_{g h} .
$$

It follows that $g \rightarrow \operatorname{Ad} U_{g} \mid \mathscr{Z}$ and $g \rightarrow \operatorname{Ad} U_{g} \mid M^{\prime}$ are homomorphisms and, if $\omega \in M \cap M^{\prime}=\mathscr{Z}, g \rightarrow \operatorname{Ad} U_{g} \mid M$ is as well; but in general, we have (2.5), with a "multiplier" Ad $\omega$.

\section{The Cocycle Condition}

The operators $U_{g}$ are associative, and as usual this leads to a "cocycle" relation:

$$
\begin{gathered}
\left(U_{g} U_{h}\right) U_{k}=\omega(g, h) U_{g h} U_{k}=\omega(g, h) \omega(g h, k) U_{g h k}, \\
U_{g}\left(U_{h} U_{k}\right)=U_{g} \omega(h, k) U_{h k}=\left[\operatorname{Ad} U_{g} \omega(h, k)\right] U_{g} U_{h k}=\left[\operatorname{Ad} U_{g} \omega(h, k)\right] \omega(g, h k) U_{g h k} .
\end{gathered}
$$

It follows that, for all $g, h, k \in G$,

$$
\omega(g, h) \omega(g h, k)=\left[\operatorname{Ad} U_{g} \omega(h, k)\right] \omega(g, h k) .
$$

This is similar to the abelian cocycle condition with left "action" $\operatorname{Ad} U_{g}$, except that the order is important, and $\operatorname{Ad} U_{g}$ is not an action of $G$ on $M$ but obeys (2.5). We say that $\omega$ is an $\operatorname{Ad} U$ cocycle if (2.5), (2.6) hold, and $\omega$ is a map $G \times G \rightarrow M$. $\omega$ unitary.

It is not clear that, given $\omega$ obeying (2.6) with respect to some automorphisms of $M$, "Ad $U_{g}$ " obeying (2.5), then there exists $U_{g} \in$ Aut $\mathscr{H}$ obeying (2.3).

\section{Equivalent Multipliers}

If $U_{g}$ and $V_{g}$ implement the automorphism $\tau$ of $\mathscr{A}$ in $\pi$, then there exists $\mu(g) \in M$ such that $V_{g}=\mu(g) U_{g}$. Conversely, if $U$ and $V$ are thus related, then $\operatorname{Ad} U \mid \pi(\mathscr{A})$ $=\operatorname{Ad} V \mid \pi(\mathscr{A})$ if either maps $\pi(\mathscr{A})$ to itself.

If so, and $U$ has multiplier $\omega$, then

$$
\begin{aligned}
V_{g} V_{h} & =\mu(g) U_{g} \mu(h) U_{h}=\mu(g)\left(\operatorname{Ad} U_{g} \mu(h)\right) \omega(g, h) U_{g h} \\
& =\mu(g)\left(\operatorname{Ad} U_{g} \mu(h)\right) \omega(g, h) \mu(g h)^{-1} V_{g h} .
\end{aligned}
$$

Hence $V_{g}$ has multiplier

$$
\omega^{\prime}(g, h)=\mu(g)\left(\operatorname{Ad} U_{g} \mu(h)\right) \omega(g, h) \mu(g h)^{-1} .
$$

Obviously, $\mu_{g}$ is unitary.

The multiplier $\omega^{\prime}(g, h)$ obeys the cocycle relation (2.6) with $\operatorname{Ad} U_{g}$ replaced by $\operatorname{Ad}\left(\mu(g) U_{g}\right)$. We say $\omega^{\prime}$ is equivalent to $\omega, \omega^{\prime} \sim \omega$, via $\mu$. This sets up an equivalence relation: $\omega \sim \omega$ via 1 ; if $\omega^{\prime} \sim \omega$ via $\mu$, then $\omega \sim \omega^{\prime}$ via $\mu^{-1}$; and if $\omega^{\prime} \sim \omega$ via $\mu$, and $\omega^{\prime \prime} \sim \omega^{\prime}$ via $\mu^{\prime}$, then $\omega^{\prime \prime} \sim \omega$ via $\mu^{\prime} \mu$. Also, $\omega \equiv 1$ is a multiplier and any $\omega \sim 1$ via $\mu$ is said to be trivial. Thus,

$$
\omega(g, h)=\mu(g)\left(\operatorname{Ad} U_{g} \mu(h)\right) \mu(g h)^{-1}
$$

is an $\operatorname{Ad}(\mu U)$-multiplier equivalent to 1 via $\mu$. One easily shows that if $(2.8)$ holds, and $\omega^{\prime}$ is equivalent to $\omega$ via $\mu^{\prime}$, then $\omega^{\prime} \sim 1$ via $\mu^{\prime} \mu$. Thus, the set of multipliers of the form (2.8) for some $\mu$ are all equivalent to each other. Any equivalence class contains an $\omega$ obeying $\omega(g, e)=\omega(e, g)=1$; we assume this relation from now on. 
We can formulate these concepts without the assumed existence of $U_{g}$; we only need an "action" $\gamma_{g}$, obeying $\gamma_{g} \in$ Aut $M$,

$$
\gamma_{g} \circ \gamma_{h}=\operatorname{Ad} \omega(g, h) \gamma_{g h},
$$

and a cocycle $\omega$ obeying

$$
\omega(g, h) \omega(g h, k)=\left[\gamma_{g} \omega(h, k)\right] \omega(g, h k) .
$$

Then equivalent multipliers, and trivial multipliers can be defined, leading to similar considerations.

If $M=\mathscr{Z}$, there is a natural multiplication between multipliers belonging to the same action $\gamma$ (which is then a homomorphism, $G \rightarrow$ Aut $M$ ), namely, the pointwise multiplication of $\omega$ as functions $G \times G \rightarrow M$. In general we can compose two projective representations as $U_{1} \otimes U_{2}$, leading to a form of product for cocycles.

Our cohomology is an example of $[4,11]$, as we show in Theorem 2 . Indeed, it is equivalent (Theorem 3). When $M$ is of type $I$, a gauge transformation can be used to reduce the multiplier to a central one, as we now show.

First, if $\pi(\mathscr{A})$ is of type $I$ with separable predual, then so is $\pi(\mathscr{A})^{\prime}=M$. Then $M$ is *-algebraically isomorphic to a $W^{*}$-algebra $M_{0}$ with abelian commutant which is then its centre, $\mathscr{Z}_{0}[13$, Theorem 5.5.11]. By the central decomposition theorem [13, Corollary 4.12.5], there exists a measure space $(Z, \mu)$ and a field of Hilbert spaces $\mathscr{H}(z)$ such that $M_{0}$ is spatially isomorphic to

$$
M_{1}=\int_{Z}^{\oplus} \mathbb{B}(\mathscr{H}(z)) d \mu(z)
$$

acting on the direct integral

$$
\mathscr{H}=\int_{Z}^{\oplus} \mathscr{H}(z) d \mu(z)
$$

The isomorphism maps $\mathscr{Z}_{0}$ onto the algebra $\mathscr{Z}_{1}$ of diagonal operators on $\mathscr{H}$. An automorphism $\gamma$ of $M$ is transferred to an automorphism $\gamma_{1}$ of $M_{1}$ leaving $\mathscr{Z}_{1}$ globally invariant. By $\left[12\right.$, p. 253, cor.], $\gamma_{1}$ is a spatial automorphism.

It is known that $\operatorname{dim} \mathscr{H}(z)$ is a measurable function of $z[12$, p. 143, Proposition 1(i)]. Thus

$$
E_{n}=\{z \in Z: \operatorname{dim} \mathscr{H}(z)=n\}
$$

defines the diagonalized projection

$$
P_{n}=\int^{\oplus} \chi_{n}(z) 1(z) d \mu(z) \in \mathscr{Z}_{1},
$$

where $\chi_{n}$ is the indicator function of $E_{n}$ and $1(z)$ is the identity operator on $\mathscr{H}(z)$. The algebras $P_{n} M_{1} P_{n}$ are homogeneous, $n \in\{1,2, \ldots \infty\}$ and are spatially isomorphic to $\mathbb{B}\left(\mathscr{H}_{n}\right) \otimes L^{\infty}\left(E_{n}, \mu\right)$; here $\operatorname{dim} \mathscr{H}_{n}=n$ and the term is missing if $\mu\left(E_{n}\right)=0$.

We now show that each $P_{n} M_{1} P_{n}$ is mapped to itself by any automorphism $\gamma_{1}$ of $M_{1}$. It is enough to show that each $P_{n}$ is invariant. Clearly, $\gamma_{1}\left(P_{n}\right)$ is a projection in $\mathscr{Z}_{1}$; let $\gamma_{1 *}\left(E_{n}\right)$ be the corresponding measurable set in $\mathscr{Z}_{1}$, where $\gamma_{1 *}$ is the pullback of $\gamma_{1} \mid \mathscr{Z}_{1}$ to $Z$. By the uniqueness theorem [12, p. 222, Theorem 4], $\mu$ and $\gamma_{1 *} \mu$ are equivalent, so $\mu\left(\gamma_{1 *} E_{n}\right) \neq 0$. Also if $m \neq n$, 


$$
\mu\left(E_{m} \cap \gamma_{1 *} E_{n}\right)=0
$$

since if not, $\mu\left(\gamma_{1 *}^{-1} E_{m} \cap E_{n}\right) \neq 0$ too, and a non-trivial part of the direct integral of dimension $n$ is mapped spatially (as $\gamma_{1}$ is spatial) onto a non-trivial part of dimension $m$. As $\mu$ is countably additive, we thus get

$$
\mu\left(\left(Z-E_{n}\right) \cap \gamma_{1 *} E_{n}\right)=0,
$$

i.e. up to sets of measure zero,

$$
\gamma_{1 *} E_{n} \subseteq E_{n}, \quad n \in\{1,2, \ldots\}, \text { showing } \gamma_{1}\left(P_{n}\right) \leqq P_{n} .
$$

Thus $M_{1}$ is the direct sum of homogeneous algebras invariant under $\gamma_{1}$. Reversing the isomorphism we see that $M$ is the direct sum of homogeneous algebras each invariant under automorphisms, in particular, under $\left\{\operatorname{Ad} U_{g}, g \in G\right\}$. It is therefore enough to consider $M$ to be a homogeneous algebra of type $I_{n}$, $1 \leqq n \leqq \infty$.

Theorem 1. Suppose $M$ is a homogeneous $W^{*}$-algebra of type I, $G$ a group and $g \rightarrow \gamma_{g}$ a map $G \rightarrow$ Aut $M$. Let $\omega: G \times G \rightarrow M$ satisfy $\left(2.5^{\prime}\right)$ and $\left(2.6^{\prime}\right)$. Then there exists a map $\mu: G \rightarrow M$ such that

$$
\omega^{\prime}(g, h)=\mu^{-1}(g) \gamma_{g}\left(\mu^{-1}(h)\right) \omega(g, h) \mu(g h)
$$

lies in $\mathscr{Z}=M \cap M^{\prime}$, and obeys the cocycle relation

$$
\omega^{\prime}(g, h) \omega^{\prime}(g h, k)=\left(\lambda_{g} \omega^{\prime}(h, k)\right) \omega^{\prime}(g, h k),
$$

where $g \rightarrow \lambda_{g}=\left.\gamma_{g}\right|_{\mathscr{Z}}$ is a homomorphism, $G \rightarrow$ Aut $\mathscr{Z}$. Moreover, if $\omega_{1}$ and $\omega_{2}$ are equivalent, then the corresponding $\omega_{1}^{\prime}, \omega_{2}^{\prime}$ are equivalent in the abelian cohomology $H^{2}(G, \mathscr{Z}, \lambda)$.

Proof. Like all automorphisms, $\gamma_{g}$ maps $\mathscr{Z}$ to itself. Since $M$ is homogeneous of type $I$, there exists a *-algebraic isomorphism $\sigma: M \rightarrow \mathscr{Z} \otimes \mathscr{B}(\mathscr{H})[12$, p. 251]. Then $\sigma \circ \gamma_{g} \circ \sigma^{-1}$ is an automorphism of $\mathscr{Z} \otimes \mathscr{B}(\mathscr{H})$ leaving the first factor invariant. Let $\lambda_{g}^{\prime}=\left(\sigma \circ \gamma_{g} \circ \sigma^{-1}\right) \mid \mathscr{Z} \otimes 1$ and put $\lambda_{g}=\sigma^{-1} \circ \lambda_{g}^{\prime} \circ \sigma$. Then $\gamma_{g} \circ \lambda_{g}^{-1}=\lambda_{g}^{-1} \circ \gamma_{g} \in$ Aut $M$, and leaves $\mathscr{Z}$ elementwise invariant. So, by [12], p. 255, or [13, p. 346], $\gamma_{g} \circ \lambda_{g}^{-1}$ is inner. So there exists $\mu_{g} \in M$ (a gauge transformation) such that $\gamma_{g} \circ \lambda_{g}^{-1}=\operatorname{Ad} \mu_{g}$. Then

$$
\begin{aligned}
\operatorname{Ad} \omega(g, h) & =\gamma_{g} \gamma_{h} \gamma_{g h}^{-1} \\
& =\left(\operatorname{Ad} \mu_{g} \lambda_{g}\right)\left(\operatorname{Ad} \mu_{h} \lambda_{h}\right)\left(\lambda_{g h}^{-1} \operatorname{Ad} \mu_{g h}^{-1}\right) \\
& =\operatorname{Ag} \mu_{g} \operatorname{Ad}\left(\lambda_{g} \mu_{h}\right)\left(\lambda_{g} \lambda_{h} \lambda_{g h}^{-1}\right) \operatorname{Ad} \mu_{g h}^{-1} \\
& =\operatorname{Ad}\left(\mu_{g}\left(\lambda_{g} \mu_{h}\right) \mu_{g h}^{-1}\right),
\end{aligned}
$$

since $\lambda_{g} \lambda_{h}=\lambda_{g h}$

Therefore,

$$
\omega(g, h)=\mu_{g}\left(\lambda_{g} \mu_{h}\right) \omega^{\prime}(g, h) \mu_{g h}^{-1}
$$

for some $\omega^{\prime}(g, h) \in \mathscr{Z}$. Then $\omega^{\prime}$ obeys $\left(2.7^{\prime}\right)$. Since $\omega$ is an $\operatorname{Ad} \mu_{g} \circ \lambda_{g}$ cocycle, the general theory ensures that $\omega^{\prime}(g, h)$ is a $\lambda_{g}$-cocycle. Thus $\omega$ is equivalent, via $\mu$ to $\omega^{\prime} \in Z^{2}(G, \mathscr{Z}, \lambda)$. Now suppose $\omega_{1}$ is equivalent to $\omega_{2}$ in the sense of non-abelian 
cohomology, so $\omega_{2} \sim \omega_{1}$ via $\mu$. Let $\omega_{1} \sim \omega_{1}^{\prime}$ via $\mu_{1}$ and $\omega_{2}^{\prime} \sim \omega_{2}$ via $\mu_{2}$, where $\omega_{1}^{\prime}$ and $\omega_{2}^{\prime} \in \mathscr{Z}^{2}(G, \mathscr{Z}, \lambda)$. Then $\omega_{2}^{\prime} \sim \omega_{1}^{\prime}$ via $\mu_{2} \mu \mu_{1}$. Hence the actions of $\omega_{1}^{\prime}$ and $\omega_{2}^{\prime}$ are related by $\operatorname{Ad}\left(\mu_{2} \mu \mu_{1}\right)$. But both have the same action, $\lambda$ : so $\lambda=\operatorname{Ad}\left(\mu_{2} \mu \mu_{1}\right) \circ \lambda$. Hence

$$
\mu_{2} \mu \mu_{1} \in \mathscr{Z}
$$

Thus $\omega_{2}^{\prime}$ and $\omega_{1}^{\prime}$ are equivalent via a central element, so

$$
\omega_{2}^{\prime} \omega_{1}^{\prime-1} \in B^{2}(G, \mathscr{Z}, \lambda) \text {. }
$$

Remark. Theorem 1 shows that there is nothing new in non-abelian cohomology in the type $I$ case. It enables us to set up cohomology groups consistently by multiplying cocycle representatives in $Z^{2}(G, \mathscr{Z}, \lambda)$. This provides an isomorphism between non-abelian cohomology with $\tau_{g} \mid \mathscr{Z}=\lambda_{g}$ given, and $H^{2}(G, \mathscr{Z}, \lambda)$. This does not mean that there is nothing new in the physics: it might not be possible to squeeze a non-abelian gauge quantum field theory into a multiplicity-free representation of the observables.

There is a connection between cocycles $\omega$ with $\omega$-action $\gamma$ and extensions of the group $G$. Let $\mathscr{U}$ denote the unitaries in $M$.

Theorem 2. The multiplication law

$$
(g, u) \circ(h, v)=\left(g h, u\left(\gamma_{g} v\right) \omega(g, h)\right)
$$

makes $G \times \mathscr{U}$ into a group $E=G \times \mathscr{U}$. Then the sequence

$$
1 \rightarrow \mathscr{U} \stackrel{i}{\longrightarrow} E \stackrel{\phi}{\longrightarrow} E / \mathscr{U} \rightarrow 1
$$

where $E / \mathscr{U} \approx G$, is exact. Moreover, for any $\omega$-representation $g \rightarrow U_{g}$ of $G$,

$$
V(g, \mu)=\mu U_{g}
$$

is a true representation of $E$. If $\omega^{\prime} \sim \omega$ via $\mu$, then the groups $G \rtimes \underset{\gamma}{ } \mathscr{U}$ and $G \underset{\gamma^{\prime}}{\rtimes} \mathscr{U}$ are isomorphic, where $\gamma_{g}^{\prime}=\operatorname{Ad} \mu_{g} \circ \gamma_{g}$, and conversely. The isomorphism is

$$
(g, u) \rightarrow\left(g, u \mu_{g}^{-1}\right) .
$$

Then the corresponding true representations of (2.11) are equal.

Proof. Elementary.

The product law (2.9) is a generalization of the semi-direct product (when $\omega=1$ ) and the central extension (when $\gamma=1 d, \mathscr{M}=\mathscr{Z}$ ).

We call (2.9) the canonical form of the extension $E$.

Theorem. Let (2.10) be an exact sequence of groups. Then there exists a pair $(\omega, \gamma)$, where $\omega$ is a 2-cocycle: $G \times G \rightarrow \mathscr{U}$ and $\gamma$ is an Ad $\omega$-homomorphism $\gamma: G \rightarrow$ Aut $\mathscr{U}$, such that

$$
E \approx \underset{\gamma}{\rtimes} \mathscr{U}
$$

with product in canonical form (2.9). 
Proof. Identify $G$ with $E / \mathscr{U}$ and to each $g \in G$ choose $e_{g} \in \phi^{-1}(g)$. Since $\phi$ is a homomorphism, $e_{g} e_{h} \in \phi^{-1}(g h)$, so there exists $\omega(g, h) \in \mathscr{U}$ such that $e_{g} e_{h}=\omega(g, h) e_{g h}$. Let $\gamma_{g}=\operatorname{Ad} e_{g} \in \operatorname{Aut} \mathscr{U}$. Then since

$$
\operatorname{Ad} e_{g} \operatorname{Ad} e_{h}=\operatorname{Ad} \omega(g, h) \circ \operatorname{Ad} e_{g h}
$$

we obtain $\left(2.5^{\prime}\right)$. Also, associativity in $G$, namely $e_{(g h) k}=e_{g(h k)}$ gives the cocycle relation $\left(2.6^{\prime}\right)$.

Finally, any element of $E$ has the unique expression $e=u e_{g}$. The product law is

$$
e_{1} e_{2}=u_{1} e_{g_{1}} u_{2} e_{g_{2}}=u_{1} \gamma_{g_{1}}\left(u_{2}\right) \omega\left(g_{1}, g_{2}\right) e_{g_{1} g_{2}}
$$

so the map

$$
u e_{g} \rightarrow(g, u)
$$

is an isomorphism in canonical form.

We see that our freedom to choose various $U_{g}$ to implement the symmetry is just choosing a representative $e_{g}$ in the coset in $E$.

\section{Energy-Momentum Anomaly}

We now give an example, the spin chain in a magnetic field, in which the space and time automorphisms commute and possess Wigner isometries, but these isometries do not commute. The anomalous commutator, which is of course a cocycle, arises in a way that is typical of anomalies the world over.

Let $\mathscr{A}$ be the spin $\frac{1}{2} C^{*}$-algebra for the linear chain, i.e. $\mathscr{A}$ is the inductive limit over finite subsets $\Lambda \subseteq Z$ of the algebras [14],

$$
\mathscr{A}(\Lambda)=\bigotimes_{j \in \Lambda} M_{2^{(j)}}, \quad|\Lambda|<\infty,
$$

where $M_{2^{(j)}}$ is a copy of $M_{2}=\mathbb{B}\left(C^{2}\right)$. The group $Z$ acts on $\mathscr{A}$ by translations:

$$
T(n) A_{j}=A_{j+n} ; \quad n, j \in Z,
$$

where $A_{j}$ is the copy of $A$ in $M_{2^{(j)}}$. Suppose the system lies in a magnetic field $\vec{M}=(0,0, M)$, so that the Hamiltonian is $H=\vec{M} \cdot \vec{S}$

$$
H=M \sum_{j \in Z}\left(\frac{1}{2} \sigma_{j}^{3}\right) .
$$

This formal infinite sum does not converge, but it defines a unique one-parameter group of automorphisms of $\mathscr{A}$, namely, those that reduce to

$$
\tau_{t}(A)=\operatorname{Ad} \exp \left(i M \sigma^{3} t / 2\right) A
$$

on each $M_{2^{(j)}}$. This action is periodic of period $4 \pi / M$, and commutes with spacetranslation. So we would say that space-translation is a symmetry in the algebraic formalism.

Each $\tau_{t}$ on $M_{2}$ has two pure stationary states $u=\left(\begin{array}{l}1 \\ 0\end{array}\right)$ and $d=\left(\begin{array}{l}0 \\ 1\end{array}\right)$. By taking infinite tensor products of these stationary states we get many invariant states of $\tau_{t}$ for the whole algebra $\mathscr{A}$. Let us choose a state that represents a magnetic domain 
wall at $j=0 \in Z$ : construct the state $[14,15]$ :

$$
\Omega=\bigotimes_{j<0} u_{j} \otimes \bigotimes_{j \geqq 0} d_{j}
$$

Then the functional

$$
\omega(A)=\langle\Omega, A \Omega\rangle, \quad A \in \mathscr{A}(\Lambda)
$$

defines by extension to the inductive limit a stationary state on $\mathscr{A}$. In the cyclic representation, say $\left(\mathscr{H}_{\omega}, \pi_{\omega}, \Omega\right)$, given by the GNS construction $[13$, p. 47$]$ timeevolution is given by a continuous one-parameter group of unitaries $\{U(t)\}_{t \in \mathbb{R}}$ on $\mathscr{H}_{\omega}$. The generator of $U(t)$ is given by (3.2) in which $\sigma_{j}^{3}$ is replaced by its representation in $\pi_{\omega}$, and a "vacuum renormalization" is made so that $H$ is convergent on a dense set in $\mathscr{H}_{\omega}$ :

$$
H=\frac{M}{2}\left(\sum_{j<0}^{-\infty}\left(\sigma_{j}^{3}-1\right)+\sum_{j \geqq 0}^{\infty}\left(\sigma_{j}^{3}+1\right)\right) .
$$

The state $\omega$ is not invariant under space-translations: the dual action $T^{*}(n)$ takes $\omega$ to $\omega_{n}$, where $\omega_{n}$ is determined by

$$
\Omega_{n}=\bigotimes_{j<n} u_{j} \otimes \bigotimes_{j \geqq n} d_{j}
$$

This might signal a spontaneous breakdown of translation invariance. However, we now show that $T(n)$ is spatial in $\pi_{\omega}$, so that each $T(n)$ is a symmetry in Wigner's sense. Nevertheless, momentum is not conserved owing to an anomaly. Define $V(n)$ on $\Omega$ by

$$
\begin{aligned}
\qquad(n) \Omega=\Omega_{n} & =\pi_{\omega}\left(\bigotimes_{j=0}^{n-1} \sigma_{j}^{1}\right) \Omega, & n>0, \\
& =\pi_{\omega}\left(\bigotimes_{j=n}^{-1} \sigma_{j}^{1}\right) \Omega, & n \leqq-1, \\
\text { where } \sigma^{1}=\left(\begin{array}{ll}
0 & 1 \\
1 & 0
\end{array}\right) . & =\Omega, \quad n=0, &
\end{aligned}
$$

Thus, $\Omega_{n}$ is the state with spin at $k$ up, $k<n$, and spin at $k$ down, $k \geqq n$. Define $V(n)$ on a general vector $\pi(\mathscr{A}) \Omega$ by

$$
V(n) \pi(A) \Omega=\pi[T(n) A] \Omega_{n} .
$$

Then $V(n)$ is unitary, obeys $V(n) V\left(n^{\prime}\right)=V\left(n+n^{\prime}\right)$, and implements $T(n)$ in $\pi$ :

$$
V(n) \pi(A) V^{-1}(n)=\pi(T(n) A) .
$$

In spite of this $(V(n)$ does not commute with $U(t)$; indeed, if $n>0$,

$$
U(t) \Omega_{n}=U(t) \pi_{\omega}\left(\sum_{k=0}^{n-1} \sigma_{k}^{1}\right) \Omega=\pi_{\omega}\left(\tau_{t}\left(\bigotimes_{k=0}^{n-1} \sigma_{k}^{1}\right)\right) \Omega
$$

(as dictated by the GNS construction)

$$
=\pi_{\omega}\left(\bigotimes_{0}^{n-1}\left(\begin{array}{cc}
0 & e^{i M t} \\
e^{-i M t} & 0
\end{array}\right)\right) \Omega=e^{i n M t} \Omega_{n} .
$$


Similarly, if $n<0$,

$$
\begin{aligned}
U(t) \Omega_{n} & =U(t) \pi\left(\bigotimes_{k=n}^{-1} \sigma_{k}^{1}\right) \Omega \\
& =\pi\left(\bigotimes_{k=n}^{-1}\left(\begin{array}{cc}
0 & e^{i M t} \\
e^{-i M t} & 0
\end{array}\right)\right) \Omega=\left(e^{-i M t)-n} \Omega_{n}\right. \\
& =e^{i n M t} \Omega_{n} .
\end{aligned}
$$

Hence $\Omega_{n}$ is an eigenstate of $H / M$ with eigenvalue $n: H / M$ is the position operator for the boundary of the domain (the boundary is only sharp for vectors of the form $\left.\Omega_{n}\right)$. In our model, there is a density for the position operator, $\frac{M}{2}\left(\sigma^{3}-1\right)$, $n<0$ and $\frac{M}{2}\left(\sigma^{3}+1\right)$ for $n \geqq 0$, in view of (3.5).

The commutation relation in Weyl form

$$
U(t) V(n)=e^{i n M t} V(n) U(t)
$$

follows immediately, at least on $\operatorname{Span}\left\{\Omega_{n}: n \in Z\right\}$. Then (3.6) must hold on all vectors: $\pi$ is irreducible, so the multiplier lies in $U(1)$ and is determined by $U(t) V(n) U^{-1}(t) U^{-1}(n)$ on any non-zero vector. Equation (3.6) gives a multiplier for the group $Z \times \hat{Z}=Z \times U(1)[16]$ : time-evolution is periodic and so forms the group $U(1)$, dual to $Z$.

It is clear that momentum is not conserved in this model. How does this "fact violation" come about? Firstly, the space-translates $\Omega_{n}$ of the stationary state $\Omega$ are different stationary states; secondly, $H$ is not positive, but has "spectral symmetry." The eigenvalue equation $H \Omega=0$ results from the "cancellation" of infinitely many $\frac{-M}{2}$ with the balancing $\frac{+M}{2}$, provided we take the infinite limit in a balanced way. This is violated on the states $\Omega_{n}$. This is exactly how the anomaly in the Virasoro algebra is described [17, p. 290], and indeed the axial anomaly also arises because the Dirac spectrum is shifted up and down by the axial gauge transformations, giving a spectral flow. In our model, the translation operator provides the spectral flow directly.

This model acts as a guide for the continuous version in Sect. 4.

\section{Energy-Momentum Anomaly in the Continuum}

We now give the continuous version of the model of Sect. 3. But it is not possible to define a continuous tensor product of the space $C^{2}$ carrying a spin $\frac{1}{2}$ representation of $U(2)$ [18]. The problem lies in the failure of the positive-definiteness of the continuous product

$$
\underset{x \in \mathbb{R}}{X}\langle\psi(x), \phi(x)\rangle=\exp \int d x \log \langle\psi(x), \phi(x)\rangle
$$

even though $\langle$,$\rangle is positive-definite. The solution to this problem is given in [19]:$ at each point $x \in \mathbb{R}$, we choose an "infinitely divisible" cyclic representation 
$\left(\mathscr{D}_{x}, \Omega_{x}\right)$, instead of the spin $\frac{1}{2}$ representation $\mathscr{D}^{1 / 2}$. For $S U(2)$, any infinitely divisible representation is an exponential of a cyclic representation [20].

To set up the continuous analogue of the model of Sect. 3, let $\lambda \in C$ and

$$
\operatorname{Exp} \lambda u=1 \oplus \lambda u \oplus \lambda^{2} \frac{u \otimes u}{\sqrt{2 !}} \oplus \ldots \in \operatorname{Exp} C^{2},
$$

where $\operatorname{Exp} C^{2}$ is the Fock space over $C^{2}$. Similarly we define $\operatorname{Exp} \lambda d$. Then

$$
\Omega_{+}=\exp \left(-|\lambda|^{2} / 2\right) \operatorname{Exp} \lambda u
$$

and

$$
\Omega_{-}=\exp \left(-|\lambda|^{2} / 2\right) \operatorname{Exp} \lambda d
$$

are unit vectors in $\operatorname{Exp} C^{2}$. Let $U \in U(2)$ and define its second quantization

$$
\operatorname{Exp} U=1 \oplus U \oplus(U \otimes U) \oplus \ldots \in \operatorname{Aut}\left(\operatorname{Exp} C^{2}\right) .
$$

Let $G$ denote the group of piecewise constant maps $U: \mathbb{R} \rightarrow U(2)$, equal to the identity outside a compact set, with pointwise multiplication. We will sometimes write $U=\bigotimes_{x} U(x)$ for the map $U: x \mapsto U(x)$.

Then we may define the continuous tensor product

$$
\mathscr{H}=\bigotimes_{x \in \mathbb{R}}\left(\operatorname{Exp} C^{2}\right)
$$

with cyclic vector

$$
\begin{array}{r}
\Omega=\underset{x}{\otimes} \Omega_{x}, \Omega_{x}=\Omega_{+}, x<0 \\
=\Omega_{-}, x>0 .
\end{array}
$$

Then (4.3) carries a representation of $G$ by the pointwise left action

$$
\left(\bigotimes_{x} U(x)\right)\left(\bigotimes_{x} \psi(x)\right)=\bigotimes_{x}(\operatorname{Exp} U(x) \cdot \psi(x))
$$

and by definition, $\mathscr{H}$ is spanned by $G$ acting on $\Omega$.

The scalar product (4.1) is then positive-definite, thus

$$
\begin{aligned}
\left\langle\underset{x}{\otimes} U_{1}(x) \Omega, \underset{x}{\otimes} U_{2}(x) \Omega\right\rangle & =\exp \int d x \log \left\langle\operatorname{Exp} U_{1}(x) \Omega_{x}, \operatorname{Exp} U_{2}(x) \Omega_{x}\right\rangle \\
& =\exp |\lambda|^{2} \int d x\left(\left\langle U_{1}(x) \omega_{x}, U_{2}(x) \omega_{x}\right\rangle_{c^{2}}-1\right),
\end{aligned}
$$

where $\omega_{x}=u, x<0$, and $\omega_{x}=d, x>0$. Let $\mathscr{A}$ be the $W^{*}$-algebra generated by the operators $\underset{x}{\otimes} U(x) \in G$ on $\mathscr{H}$. Certain automorphisms of $\mathscr{A}$ are spatial. Naturally, conjugation by elements of $G$, being inner, are spatial. More, let $U_{1}=\bigotimes_{x} U_{1}(x)$ be such that, outside a compact set $K, U_{1}$ leaves the states defined by $u, d$ invariant, i.e. $U_{1}$ is a rotation about the third axis. Suppose $U_{1}$ is piecewise constant in $K$. Then the automorphism

$$
U \mapsto \otimes_{x} U_{1}(x) U(x) U_{1}^{-1}(x)
$$


is a spatial automorphism. Thus, let $\tau_{t}$ be the automorphism of $\mathscr{A}$ due to a constant magnetic field $M$ in the third direction. On $G$, this reduces to (4.7) with $U_{1}(x)=\left(\begin{array}{cc}e^{i t M / 2} & 0 \\ 0 & e^{-i t M / 2}\end{array}\right) \forall x$. Then $\Omega$ defines a state on $\mathscr{A}$ invariant under $\tau_{t}$, which is therefore implemented, by $V(t)$ say, given by

$$
V(t)(\underset{x}{\otimes} U(x) \Omega)=\underset{x}{\otimes} \tau_{t}^{0}(U(x)) \Omega_{x},
$$

where $\tau_{t}^{0}=\operatorname{Ad} U_{1}(x)$. Then $V\left(t_{1}\right) V\left(t_{2}\right)=V\left(t_{1}+t_{2}\right)$, and $V(t+4 \pi / M)=V(t)$. The group of space translations acting on $G$ namely $T_{a} \underset{x}{\otimes} U(x)=\underset{x}{\otimes} U(x-a)$ is also spatial. I claim that $T_{a}$ is implemented by $W(a)$ :

$$
W(a) \underset{x}{\otimes} U(x) \Omega=\bigotimes_{x}^{\otimes} U(x-a) \Omega_{a},
$$

where $\Omega_{a}$ has the wall of the magnetic domain at $a$ instead of at 0 :

$$
\Omega_{a}=\bigotimes_{-\infty}^{a} \Omega_{+} \otimes \bigotimes_{a}^{\infty} \Omega_{-} .
$$

We must justify (4.9) by showing that it lies in $\mathscr{H}$. In the spin $\frac{1}{2}$ representation of $U(2)$, the element $\sigma^{1}=\left(\begin{array}{ll}0 & 1 \\ 1 & 0\end{array}\right)$ converts $\lambda u$ to $\lambda d$ and vice versa. It follows that $\operatorname{Exp} \sigma^{1}$ takes $\operatorname{Exp} \lambda u$ to $\operatorname{Exp} \lambda d$ in $\operatorname{Exp} C^{2}$, and vice versa. Therefore the element of $\mathscr{A}$, by (4.5)

$$
F_{a, b}=\underset{x \in[a, b]}{\otimes} \sigma_{x}^{1}
$$

takes $\underset{x \in[a, b]}{\otimes}(\operatorname{Exp} \lambda u)_{x}$ to $\underset{x \in[a, b]}{\otimes}(\operatorname{Exp} \lambda d)_{x}$ and vice versa. Thus

$$
\begin{aligned}
\Omega_{a} & =\mathrm{F}_{0, a} \Omega, & & \mathrm{a}>0, \\
& =\mathrm{F}_{a, 0} \Omega, & & \mathrm{a}<0,
\end{aligned}
$$

lies in $\mathscr{H}$. It is then trivial to show that $W(a)$ is unitary on $\mathscr{H}$, and $W\left(a_{1}\right) W\left(a_{2}\right)$ $=W\left(a_{1}+a_{2}\right)$.

As in the discrete model, Sect. 3, space and time automorphisms commute: $\tau_{t} T_{a}=T_{a} \tau_{t}$; but their implementing operators $V(t), W(a)$ do not commute. We can now compute the multiplier of the representation of the group $\mathbb{R}^{2}$ given by $U(a, t)$ $=W(a) V(t)$. We get

$$
\begin{aligned}
& U\left(a_{1}, t_{1}\right) U\left(a_{2}, t_{2}\right) A \Omega=W\left(a_{1}\right) V\left(t_{1}\right) W\left(a_{2}\right) \tau_{t_{2}} A \Omega \\
& \quad=W\left(a_{1}\right) V\left(t_{1}\right)\left(T_{a_{2}} \tau_{t_{2}} A\right) \Omega\left(a_{2}\right)=W\left(a_{1}\right)\left(T_{a_{2}} \tau_{t_{1}+t_{2}} A\right) V\left(t_{1}\right) \Omega\left(a_{2}\right) \\
& \quad=W\left(a_{1}\right)\left(T_{a_{2}} \tau_{t_{1}+t_{2}} A\right)\left(\tau_{t_{1}} \underset{x \in\left[0, a_{2}\right]}{\otimes} \sigma_{x}^{1}\right) \Omega \text { if } a_{2}>0 .
\end{aligned}
$$

Now, $\tau_{t} \underset{x \in[0, a]}{\otimes} \sigma_{x}^{1}=\bigotimes_{x \in[0, a]} \tau_{x}^{0} \sigma_{x}^{1}$ and $\tau_{t}^{0} \sigma^{1}=\left(\begin{array}{cc}0 & e^{i M t} \\ e^{-i M t} & 0\end{array}\right)$. This multiplies $u$ by $e^{-i M t}$. In the interval $\left[0, a_{2}\right]$ the second quantization of this is

$$
e^{i M t N\left[0, a_{2}\right]}=\operatorname{Exp}\left(e^{i M t x\left[0, a_{2}\right]}\right),
$$


where $N[a, b]$ is the number operator in the interval $[a, b]$. Hence

$$
\begin{aligned}
& U\left(a_{1}, t_{1}\right) U\left(a_{2}, t_{2}\right) A \Omega=W\left(a_{1}\right)\left(T_{a_{2}} \tau_{t_{1}+t_{2}} A\right) e^{-i M t_{1} N\left[0, a_{2}\right]} \Omega\left(a_{2}\right) \\
& \quad=\left(T_{a_{1}+a_{2}} \tau_{t_{1}+t_{2}} A\right) e^{-i M t_{1} N\left[a_{1}, a_{1}+a_{2}\right]} \Omega\left(a_{1}+a_{2}\right) .
\end{aligned}
$$

Since

$$
U\left(a_{1}+a_{2}, t_{1}+t_{2}\right) A \Omega=\left(T_{a_{1}+a_{2}} \tau_{t_{1}+t_{2}} A\right) \Omega\left(a_{1}+a_{2}\right)
$$

we see that the multiplier is

$$
\omega\left(a_{1}, t_{1} ; a_{2}, t_{2}\right)=e^{-i M t_{1} N\left[a_{1}, a_{1}+a_{2}\right]}, \quad a_{2}>0 .
$$

For $a_{2}<0$ we get the same formula if we interpret $N[a, b]=-N[b, a]$. The action of the group $\mathbb{R}^{2}$ on the cocycle is

$$
\operatorname{Ad} U(a, t) \omega\left(a_{1}, t_{1}, a_{2}, t_{2}\right)=\omega\left(a_{1}+a, t_{1} ; a_{2}+a, t_{2}\right)
$$

One verifies that, with this action, $\omega$ obeys the cocycle relation (2.6). In this case, $M=\mathscr{Z}$ is abelian, being the $W^{*}$-algebra generated by the number density, i.e. by $\{\exp i N[a, b], a<b \in \mathbb{R}\}$.

We have an operator anomaly, albeit an abelian one. Heuristically, this arises as follows. The local currents, which generate $G$, are given by $\vec{J}(x)=\frac{1}{2} a_{i}^{*}(x) \vec{\sigma}_{i j} a_{j}(x)$, where $a_{i}^{*}(x), i=1,2$ create the two spin-state $u, d$ in Fock space. The naive formula for the energy in a magnetic field in the third direction, $M \int_{-\infty}^{\infty} J^{3}(x) d x$, diverges on $\Omega$; we must subtract half the number density, $N(x) / 2, x<0$ and add $N(x) / 2, x>0$, to remove the zero point energy. Then the "renormalized" Hamiltonian

$$
H=M \int_{-\infty}^{0}\left(J(x)-\frac{N(x)}{2}\right) d x+\int_{0}^{\infty}\left(J(x)+\frac{N(x)}{2}\right) d x
$$

annihilates $\Omega$ and makes sense on the states of $\mathscr{H}$. But it fails to commute with space-translation:

$$
W(a) H-H W(a)=-\int_{0}^{a} M N(x) d x=-M N[0, a],
$$

giving the anomaly, again caused by spectral flow.

By choosing $a=t$, we obtain a projective unitary representation of $\mathbb{R}: t \rightarrow U(t, t)$ not reducible to a true representation.

We can turn this model round, and choose $\mathscr{Z}$ as the algebra of observables, and $\mathscr{A}$ as the non-abelian gauge group. The observables then are generated by the number operator density, which is invariant under the "time-evolution" $\tau_{t}$ given by the external constant gauge field in the third direction, which (being the identity automorphism) commutes with space-translation. Let us look at this system in the state $\Omega$, which has a kink in its gauge potential at $x=0$. We find that the trivial action of the time evolution, $\tau_{t}$ on $\mathscr{Z}$, is non-trivially represented in the Hilbert space $\mathscr{H}$ built on $\Omega$, namely, it is given by $V(t)$ ! A shocking violation of fact. 


\section{References}

1. Wigner, E.P.: Gruppentheorie und ihre Anwendung. Braunschweig: Wieweg 1931 (English translation: New York: Academic Press 1959

2. Wigner, E.P.: On Unitary Representations of the Inhomogeneous Lorentz Group. Ann. Math. 40, 149-204 (1939)

3. Bargmann, V.: A note on Wigner's theorem on symmetry operations. J. Math. Phys. 5, 862-868 (1964)

4. Eilenberg, S., MacLane, S.: Cohomology theory in abstract groups. I. Ann. Math. 48, 51-78 (1947). II lbid, 326-341

5. Bargmann, V.: On unitary ray representations of continuous groups. Ann. Math. 59, 1-46 (1954)

6. Streater, R.F., Wightman, A.S.: PCT, spin and statistics and all that. Reading, MA: Addison Wesley, 3rd Edition, 1989, p. 6

7. Bardeen, J., Cooper, L.N., Schrieffer, J.R.: Theory of superconductivity. Phys. Rev. 108, 1175-1204 (1975)

8. Nambu, Y., Jona-Lasinio, G.: Dynamical model of elementary particles based on an analogy with superconductivity, I. Phys. Rev. 122, 345-358 (1961); J. Goldstone: Ph. D. Thesis, Cambridge (1959)

9. Streater, R.F.: Spontaneously broken symmetry in axiomatic theory. Proc. R. Soc. A 287, 510-518 (1965)

10. Y-Hong Chen, Wilczek, F., Witten, E., Halperin, B.I.: On anyon superconductivity. Int. J. Mod. Phys. B 3, 1001-1067 (1989)

11. Lue, A.: Cohomology of groups relative to a variety. J. Algebra 69, 155-174 (1981)

12. Dixmier, J.: Les algèbres d'opérateurs dans l'espace hilbertien. Paris: Gauthier-Villars 1957

13. Pedersen, G.K.: $C^{*}$-algebras and their automorphism groups. London, New York: Academic Press 1979

14. Streater, R.F.: The Heisenberg ferromagnet as a quantum field theory. Commun. Math. Phys. 6, 233-247 (1967);

Streater, R.F.: A Continuum Analogue of the Lattice Gas. Commun. Math. Phys. 12, 226-232 (1969)

15. Streater, R.F.: Fields and particles; Inaugural Lecture, Bedford College, 1972. Dillon's Bookshop, Gower Place, London

16. Mackey, G.W.: Theory of unitary group representations, Chicago, IL: University of Chicago Press 1976

17. Schwarz, J.H.: Dual resonance theory. Phys. Rep. 8C, 270-335 (1973)

18. Dubin, D.A., Streater, R.F.: Non-existence of the continuum ferromagnet. Nuovo Cimento 50, 154-157 (1967)

19. Streater, R.F.: Current commutation relations, continuous tensor products and infinitely divisible group representations. In: Local Quantum Theory, Jost, R. (ed.) New York: Academic Press 1969

20. Araki, H.: Factorizable representations of current algebras. Publ. RIMS (Kyoto) 5, 361-422 (1970), Theorem 7.1

Communicated by A. Jaffe

Received April 4, 1990 
